\title{
EQUIDIMENSIONALITY OF LAGRANGIAN FIBRATIONS ON HOLOMORPHIC SYMPLECTIC MANIFOLDS
}

\author{
Daisuke Matsushita
}

\begin{abstract}
We prove that every irreducible component of every fibre of Lagrangian fibrations on holomorphic symplectic manifolds is a Lagrangian subvariety. Especially, Lagrangian fibrations are equidimensional.
\end{abstract}

\section{Introduction}

We begin with the definition of Lagrangian subvarieties .

Definition 1. Let $X$ be a complex manifold with a holomorhpic symplectic form $\omega$. A subvariety $Y$ is said to be a Lagrangian subvariety if $\operatorname{dim} Y=\left(\frac{1}{2}\right) \operatorname{dim} X$ and there exists a resolution $\nu: \tilde{Y} \rightarrow Y$ such that $\nu^{*} \omega$ is identically zero on $\tilde{Y}$.

Note that this notion does not depend on the choice of $\nu$. We prove the following theorem.

Theorem 1. Let $X$ be a Kähler manifold and $f: X \rightarrow B$ a proper surjective morphism over a normal variety $B$. Assume that there exists a $d$-closed holomorphic symplectic form $\omega$ on $X$ and a general fiber of $f$ is a Lagrangian subvariety with respect to $\omega$. Then every irreducible component of every fibre of $f$ is a Lagrangian subvariety. Especially $f$ is equidimensional.

Since every holomorphic form on a compact Kähler manifold is $d$-closed, we obtain the following result from combining Theorem 1 with [2, Theorem 2] and [3, Theorem 1].

Corollary 1. Let $f: X \rightarrow B$ be a surjective morphism from an irreducible symplectic manifold $X$ to a normal projective variety $B$. Assume that $0<$ $\operatorname{dim} B<\operatorname{dim} X$. Then every irreducible component of every fibre of $f$ is a Lagrangian subvariety.

Remark. If we drop the condition of properness, then $f$ is not necessarily equidimensional. Let $f$ be a morphism from $\mathbb{C}^{4}$ to $\mathbb{C}^{2}$ defined by

$$
f(x, y, z, w):=(x, x y)
$$

Received February 10, 2000.

1991 Mathematics Subject Classification. Primary 14E40, Secondary 14D05.

*Research Fellow of the Japan Society for the Promotion of Science. 
and $\omega:=d x \wedge d z+d y \wedge d w$. Then $\omega$ is a $d$-closed holomorphic symplectic form and a general fibre of $f$ is a Lagrangian subvariety with respect to $\omega$. Since $\operatorname{dim} f^{-1}(0)=3, f$ is not equidimensional.

From Theorem 1, we obtain some information of the singularities of $B$.

Corollary 2. For every point $p$ of $B$, there exists a Stein neighborhood $U$ of $p$ and a finite morphism $\pi: \tilde{U} \rightarrow U$ from a smooth Stein manifold $\tilde{U}$.

Proof. For a point $p$ of $B$, we choose a point $q \in f^{-1}(p)$ and a smooth Stein neighborhoods $W$ of $q$. Since $f$ is equidimensional, we obtain a finite morphism $\pi: \tilde{U} \rightarrow U$ from a smooth Stein manifold $\tilde{U}$ by cutting $W$ with hypersurfaces.

Remark. The author does not know whether there exists an example such that $B$ is not smooth.

\section{Proof of Theorem 1}

We refer the following theorem due to Kollár [1, Theorem 2.2] and Mo. Saito [4, Theorem 2.3, Remark 2.9].

Theorem 2. Let $f: X \rightarrow B$ be a proper surjective morhpism from a smooth Kähler manifold $X$ to a normal variety $B$. Then $\mathrm{R}^{i} f_{*} \omega_{X}$ is torsion free, where $\omega_{X}$ is the dualizing sheaf of $X$.

Proof. Let $\bar{\omega}$ be the complex conjugate of $\omega$. Since $\omega$ is $d$-closed, $\bar{\omega}$ can be considered as an element of $H^{2}\left(X, \mathcal{O}_{X}\right)$. By Leray spectral sequence, there exists a morphism

$$
H^{2}\left(X, \mathcal{O}_{X}\right) \rightarrow H^{0}\left(B, \mathrm{R}^{2} f_{*} \mathcal{O}_{X}\right)
$$

Then $\bar{\omega}$ is a torsion element in $H^{0}\left(B, \mathrm{R}^{2} f_{*} \mathcal{O}_{X}\right)$ since a general fibre of $f$ is a Lagrangian subvariety. In addition, $\omega_{X} \cong \mathcal{O}_{X}$. Hence $\bar{\omega}$ is zero in $H^{0}\left(B, \mathrm{R}^{2} f_{*} \mathcal{O}_{X}\right)$ by Theorem 2. We derive a contradiction assuming that there exists an irrducible component of a fibre of $f$ which is not a Lagrangian subvariety. The letter $V$ denotes an non Lagrangian component. We take an embedding resolution $\pi: \tilde{X} \rightarrow X$ of $V$. Let $\tilde{V}$ be the proper transform of $V$. We will show that $\pi^{*} \omega$ is not zero in $H^{0}\left(\tilde{V}, \Omega_{\tilde{V}}^{2}\right)$. If $\operatorname{dim} V=(1 / 2) \operatorname{dim} X$, it is obious by the definition. If $\operatorname{dim} V>(1 / 2) \operatorname{dim} X$, we take a smooth point $q \in V$ such that $\pi$ is isomorhpic in a neighborhood of $q$. Since $\operatorname{dim} V>(1 / 2) \operatorname{dim} X$ and $\omega$ is nondegenerate, the restriction of $\omega$ on the tangent space of $V$ at $q$ is nonzero. Because $\pi$ is isomorphic in a neighborhood of $q, \pi^{*} \omega$ is not zero in $H^{0}\left(\tilde{V}, \Omega_{\tilde{V}}^{2}\right)$. Take the complex conjugate, $\pi^{*} \bar{\omega}$ is not zero in $H^{2}\left(\tilde{V}, \mathcal{O}_{\tilde{V}}\right)$. Therefore $\bar{\omega}$ is not zero in $H^{2}\left(V, \mathcal{O}_{V}\right)$. Let $p:=f(V)$ and $X_{p}:=f^{-1}(p)$. We consider the following morphism:

$$
\mathrm{R}^{2} f_{*} \mathcal{O}_{X} \otimes k(p) \rightarrow H^{2}\left(X_{p}, \mathcal{O}_{X_{p}}\right) \rightarrow H^{2}\left(V, \mathcal{O}_{V}\right)
$$

Then $\bar{\omega}$ is zero in $\mathrm{R}^{2} f_{*} \mathcal{O}_{X} \otimes k(p)$ and nonzero in $H^{2}\left(V, \mathcal{O}_{V}\right)$. That is a contradiction. 


\section{Acknowledgment}

The author express his thanks to Professors A. Beauville, A. Fujiki, Y. Miyaoka, S. Mori and N. Nakayama for their advice and encouragement.

\section{References}

[1] J. Kollár, Higher direct images of dualizing sheaves, Ann. of Math. 123 (1986), 11-42.

[2] D. Matsushita, On fibre space structures of a projective irreducible symplectic manifold, Topology 38 (1999), 79-83.

[3] D. Matsushita, Addendum to: On fibre space structures of a projective irreducible symplectic manifold, to appear in Topology.

[4] M. Saito, Decomposition theorem for proper Kähler morphisms, Tôhoku Math. J. 42 (1990), 127-148.

Research Institue for Mathematical Sciences, Kyoto University, Oiwake-Cho KiTASHIRAKAWA, SAKYO-KU KYOTO 606-8052 JAPAN

E-mail address: tyler@kurims.kyoto-u.ac.jp 